\title{
Effects of ion bombardment on interband cascade laser structures
}

C. Merritt, C. Kim, M. Kim, C. Canedy, W. Bewley, et al.

C. D. Merritt, C. S. Kim, M. Kim, C. L. Canedy, W. W. Bewley, M. V. Warren, I. Vurgaftman, J. R. Meyer, "Effects of ion bombardment on interband cascade laser structures," Proc. SPIE 11288, Quantum Sensing and Nano Electronics and Photonics XVII, 112881N (31 January 2020); doi: 10.1117/12.2545898

SPIE. Event: SPIE OPTO, 2020, San Francisco, California, United States 


\title{
Effects of ion bombardment on interband cascade laser structures
}

\author{
C. D. Merritt, ${ }^{a}$ C. S. Kim, ${ }^{\mathrm{a}}$ M. Kim, ${ }^{\mathrm{b}}$ C. L. Canedy, ${ }^{\mathrm{a}}$ W. W. Bewley, ${ }^{\mathrm{a}}$ M. V. Warren, ${ }^{\mathrm{a}, \mathrm{c}}$ \\ I. Vurgaftman, ${ }^{a}$ and J. R. Meyer*a \\ ${ }^{a}$ Code 5613, Naval Research Laboratory, Washington, DC 20375 \\ bJacobs, 7740 Milestone Parkway, Suite 150, Hanover MD 21076 \\ cCurrently: Raytheon Integrated Defense Systems, 362 Lowell, Andover MA 01810
}

\begin{abstract}
We report a preliminary investigation of ion bombardment (IB) effects on interband cascade laser (ICL) properties. Under some conditions, IB almost completely suppresses the vertical transport through a broad-area laser, although other times only a partial or negligible suppression is observed. To elucidate the mechanism that induces the suppression and in what part of the structure it occurs, we investigated the effects of IB on samples containing only ICL sub-regions. While IB increased the resistivity of a lightly- $n$-doped GaSb layer such as that used as a top or bottom separate confinement layer in an ICL, that layer was still much too conductive to strongly suppress the current flowing through a full device. The voltage drop was larger following IB of an InAs-AlSb superlattice such as that used in the top and bottom optical cladding layers in an ICL, although the effect was not large enough to fully account for the strong net suppression. And finally, the resistivity of an interband cascade LED containing the same active stages as an ICL was actually found to decrease following IB. Despite the inconclusive and sometime inconsistent findings of this study, it is nonetheless clear that if the effects can be controlled reproducibly, IB may provide a valuable tool for enhancing such ICL device configurations as weakly-index-guided narrow ridges and interband cascade vertical-cavity surface-emitting laser mesas that inject current and emit light only within a small central aperture.
\end{abstract}

Keywords: Interband cascade laser, mid-infrared, ion bombardment, weak index guiding, vertical-cavity surface-emitting laser

\section{INTRODUCTION}

In recent years, the type-II interband cascade laser (ICL) has become a dominant coherent source for applications such as chemical absorption spectroscopy in the mid-wave infrared (mid-IR) spectral band spanning 3-6 $\mu \mathrm{m}$ [1]. It combines a relatively long upper-level lifetime, characteristic of semiconductor interband transitions, with the voltage-efficient cascading scheme originally introduced for intersubband-based quantum cascade lasers (QCLs). Electrons and holes recombine to provide gain in each stage of the ICL's cascaded active region, even though the contacts inject and remove only electrons. Following the first room-temperature (RT) continuous wave (cw) operation in 2008 [2], ICLs have operated in cw mode up to $118{ }^{\circ} \mathrm{C}$ [3], produced $>500 \mathrm{~mW}$ of cw power at RT [4], displayed wallplug efficiencies up to $18 \%$ for RT cw operation [5], and operated in cw mode at RT to wavelengths as long as $6 \mu \mathrm{m}$ [6,7]. The low ICL drive power [8], which is 1-2 orders of magnitude less than for a QCL, is especially important in applications such as portable battery- or solar-powered laser spectroscopy systems that require a small footprint and low power budget. The following sub-sections briefly overview some other recent advances.

\subsection{Single-Mode ICLs}

Distributed-feedback (DFB) ICLs emitting in a single spectral mode have generated up to $55 \mathrm{~mW}$ of cw power at RT [9], and have operated at wavelengths as long as $6.6 \mu \mathrm{m}$ [6]. Nanoplus currently offers DFB ICL products that span wavelengths from 3 to $6 \mu \mathrm{m}$ [10]. Thorlabs Quantum Electronics recently reported a novel narrow ridge architecture [11] that alternates regions with corrugated sidewalls (to suppress higher-order lateral modes) [12] with regions having a top DFB grating. At $\lambda=3.3 \mu \mathrm{m}$, the resulting lasers generated up to $42 \mathrm{~mW}$ of $\mathrm{cw}$ power at RT in a single spectral mode. DFB ICLs are now used in numerous chemical sensing systems [13-16].

*mwir_laser@nrl.navy.mil; (202) 767-3276

Quantum Sensing and Nano Electronics and Photonics XVII, edited by Manijeh Razeghi,

Jay S. Lewis, Giti A. Khodaparast, Pedram Khalili, Proc. of SPIE Vol. 11288, 112881N

(c) 2020 SPIE · CCC code: $0277-786 X / 20 / \$ 21 \cdot$ doi: $10.1117 / 12.2545898$ 


\subsection{Interband Cascade Vertical Cavity Surface Emitting Lasers (ICVCSELs)}

NRL recently demonstrated ICVCSELs operating at $\lambda \approx 3.4 \mu \mathrm{m}$ [17]. The bottom distributed Bragg reflector (DBR) mirror was formed by 22.5 repeats of alternating GaSb and AlAsSb layers, the cavity included 15 interband cascade stages split into three groups positioned at the antinodes of the optical field, and the top mirror was formed by a 4-period Ge/ $/ \mathrm{Al}_{2} \mathrm{O}_{3}$ DBR. The ICVCSELs operated in pulsed mode to $70{ }^{\circ} \mathrm{C}$, with circularly-symmetric outputs and RT threshold current densities as low as $390 \mathrm{~A} / \mathrm{cm}^{2}$. However, the differential slope efficiencies were low due to loss in the top and bottom mirrors, and mode overlap with the annular top contact metal. The smallest device (20 $\mu \mathrm{m}$ aperture diameter) operated in a single spectral mode. The University of Montpellier is also now developing ICVCSELs [18]. Although a mid-IR VCSEL reported by the Walter Schottky Institut recently operated $\mathrm{cw}$ to $-7^{\circ} \mathrm{C}$ for emission at $\lambda=4.0 \mu \mathrm{m}$, their diode structure employed multiple type-II "W" quantum wells rather than interband cascade stages [19].

\subsection{Interband Cascade Light Emitting Devices (ICLEDs)}

NRL has also demonstrated ICLEDs [20], which for emission at a peak wavelength of $\lambda \approx 3.2 \mu \mathrm{m}$ displayed higher maximum output powers, radiances, and efficiencies than any earlier mid-IR LEDs. A novel design that positioned 22 active stages at antinodes of the optical field reflected by the metal mirror produced up to $2.9 \mathrm{~mW}$ cw from a $400 \mu \mathrm{m}$ diameter circular mesa at RT [21]. Subsequent devices emitted $>1.3 \mathrm{~mW}$ cw at a peak wavelength of $4.1 \mu \mathrm{m}$ when operated at RT [22]. Most recently, Nanoplus and University of Würzburg reported $>5 \mathrm{~mW}$ of cw output from a $640 \times 640 \mu^{2}$ square mesa with $0.7 \%$ WPE [23].

\subsection{Interband Cascade Laser Frequency Combs}

Jet Propulsion Laboratory and NRL recently demonstrated the first ICL frequency combs [24]. The inherent nonlinearity of the ICL medium supports self-starting broadband comb generation with low electrical power consumption $(<1 \mathrm{~W})$ at RT. Furthermore, ICL combs with sub-MHz free-running optical linewidth yielded dual-comb spectroscopy (DCS) in the 3-4 $\mu \mathrm{m}$ region [25]. A team led by TU Wien subsequently reported a monolithic platform [26] that combines an ICL frequency comb with an interband cascade detector (ICD) [27].

\subsection{Photonic Integrated Circuits Incorporating Interband Cascade Lasers on Silicon and III-V Platforms}

Mid-IR photonic integrated circuits (PICs) compatible with existing Si photonic and electronic technologies promise to enable low-cost, compact sensing systems. NRL, UCSB, and U. Wisconsin previously reported the heterogeneous integration on Si of Fabry-Perot and DFB QCLs operating at $4.8 \mu \mathrm{m}$ [28]. UCSB and NRL more recently demonstrated the integration of ICLs emitting at $\lambda \approx 3.6 \mu \mathrm{m}$ on silicon-on-insulator (SOI) waveguides [29]. In pulsed mode, an 11- $\mu \mathrm{m}-$ wide III-V mesa on top of a 1- $\mu \mathrm{m}$-wide silicon waveguide emitted $>6 \mathrm{~mW}$ at RT [30], and operated to $50{ }^{\circ} \mathrm{C}$ even though most of the injected current was lost to sidewall leakage that resulted from non-optimal processing.

Whereas silicon-based PICs may ultimately span uv to longwave IR spectral bands, some applications that do not require multi-spectral operation may benefit from the simpler and more straightforward processing of devices on the native III-V substrates. NRL recently proposed a flexible III-V PIC architecture that flexibly combines ICLs, ICDs, passive waveguides, and other optical components on a GaSb platform, or analogous QCL-based devices on an InP platform [31]. Obvious applications include the sensing of trace gases such as methane, carbon dioxide, carbon monoxide, formaldehyde, etc., in ambient air, and on-chip beam combining for power scaling.

\section{WEAK INDEX GUIDING}

It is well known that a ridge-waveguide laser can remain single mode (and display enhanced brightness) for wider ridges when the etch that defines the lasing mode is shallow [32,33], i.e., stopping above the separate-confinement regions and active gain QWs in the case of an ICL. This weakly confines the optical mode, whereas a deep etch (stopping below the separate-confinement regions and/or active QWs) induces a larger lateral index step so that additional guided modes are present. Figures 1(a) and 1(b) illustrate the two cases of strong and weak index guiding, respectively. Most of the ICLs to date have employed strong index guiding, since a shallow etch would allow the injected current to spread extensively into 
the outer ridge [26], severely limiting the efficiency of the double ridge geometry. This contrasts the case of GaAs- or InPbased diode lasers emitting in the near IR, which are far less susceptible to current spreading because their electrical conductivity is nearly isotropic. Weakly-index-guided ICLs have rarely been fabricated for this reason, and the few exceptions have suffered from lower wallplug efficiency (WPE) [34]. All of the high-power ICLs demonstrated to date have employed strong index guiding that resulted from a deep etch like that illustrated in Fig. 1(a) to prevent lateral current spreading [4,5].

Naturally, a weakly-index-guided ICL could maintain high WPE if it were possible to somehow suppress the parasitic flow of current into the outer portion of the double ridge shown in Fig. 1(b). Various other semiconductor device technologies have used ion bombardment (IB) to deplete carriers, and thereby substantially reduce the electrical conductivity in analogous geometries [35,36]. On the assumption that a similar suppression can be realized in ICLs, Fig. 1(c) illustrates a hypothetical weakly-index-guided geometry that could maintain high efficiency because current flows only within the inner ridge that provides gain.

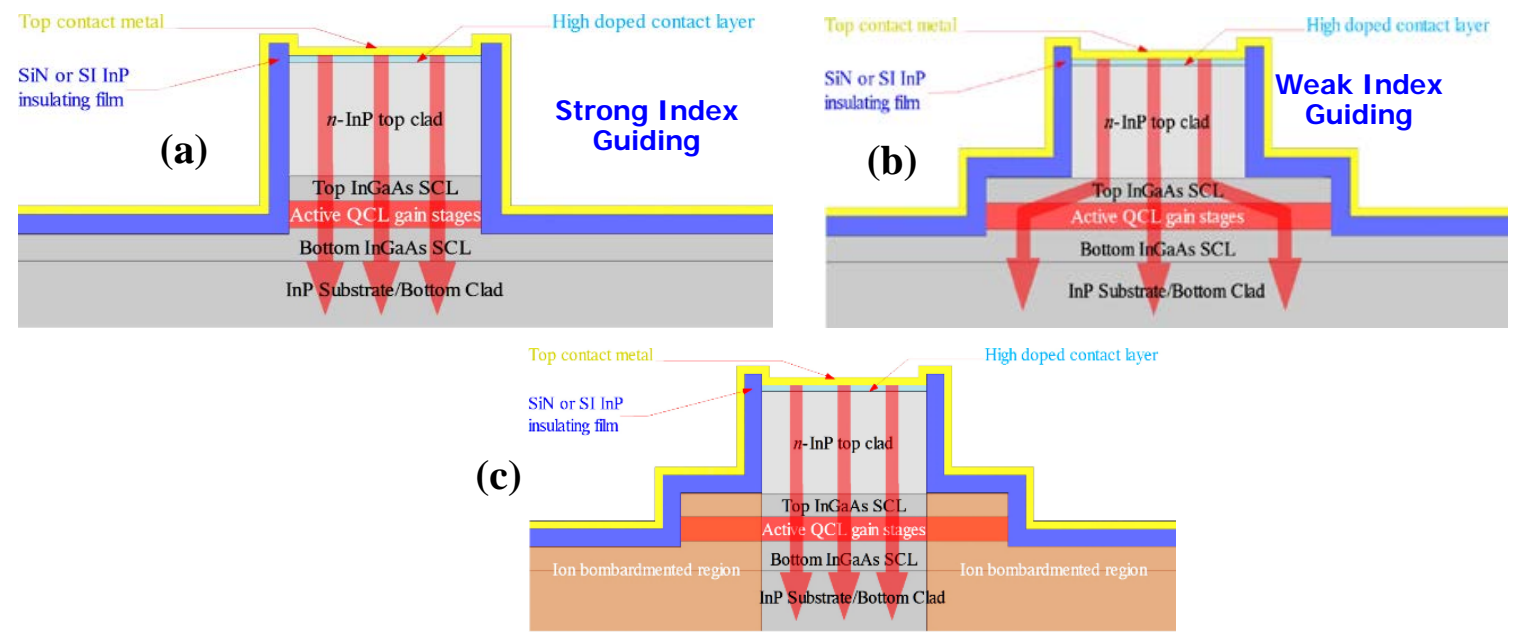

Figure 1. Schematic of ICL ridges with: (a) strong index guiding; (b) weak index guiding by etching a double ridge, but with low WPE due to current spreading into the outer ridge; and (c) weak index guiding with ion bombardment of the outer ridge that could hypothetically minimize current spreading.

In fact, the index-guided ICL of Fig. 1(c) may exhibit higher efficiency than the gain-guided structure of Fig. 1(a), due to much lower scattering loss at the ridge sidewalls. In order to maintain a single lateral mode with good beam quality, a strongly-index-guided ICL ridge must be relatively narrow, which causes the optical mode to overlap the ridge sidewalls. Because the etched surfaces are never perfectly smooth, scattering loss induced by the sidewall roughness inevitably lowers the laser efficiency. Due to this effect, the WPEs of strongly-index-guided ICLs have always decreased with decreasing ridge width. However, the index-guided ICL of Figs. 1 would experience far less sidewall scattering because: (1) the larger optimal ridge width leads to lower overlap of the optical mode with the sidewalls; and (2) for any given inner ridge width, the much weaker index contrast at its boundary will induce far less scattering due to any roughness present at that boundary.

Another factor that often compromises the efficiency of gain-guided ICLs is parasitic current leakage at the ridge sidewalls, especially if the processing with reactive ion etching (RIE) and photo-enhanced chemical vapor deposition (PECVD) of the dielectric is sub-optimal (i.e., sidewall leakage often lowers the processing yield). A weakly-index-guided configuration would substantially reduce sidewall leakage and improve the processing yield, since no leakage occurs at the inner ridge boundary (for which the etch does not reach the active stages), and the outer boundary is much more remote. Furthermore, a gentler wet etch may be used to etch the outer ridge, since in the absence of optical overlap the sidewall angle is unimportant. This contrasts the strongly-index-guided configuration for which the sidewalls must be nearly vertical to keep the ridge narrow. The primary disadvantage of weak index guiding may be less effective lateral heat dissipation, since additional semiconductor material will separate the active core from the sidewall metallization that overcoats an insulating dielectric such as SiN. 
Motivated by these potential advantages and the successful suppression of parasitic currents in other material systems, we carried out the first investigation of IB effects on ICL structures to be reported. While a clear and consistent picture of how IB affects the properties has yet to emerge, some of the findings are of considerable interest.

\section{ION BOMBARDMENT OF THE FULL ICL}

We found in an early study that while irradiation with a moderate dose of protons strongly suppresses the light emission from type-II InAs-GaInSb quantum wells (presumably because Shockley-Read-Hall (SRH) defects are generated), the effect on lateral current flow was marginal. However, we demonstrated more recently that bombardment by a sufficient dose of protons can effectively eliminate the vertical transport through a broad-area ICL up to the bias voltages of interest. In that experiment, each broad-area ridge of width $150 \mu \mathrm{m}$ and length $2 \mathrm{~mm}$ was irradiated by a uniform sheet density of protons with energy of $340 \mathrm{keV}$. The results shown in Fig. 3(a) indicate that while a dose $\leq 6 \times 10^{12} \mathrm{~cm}^{-2}$ had little effect on the $I-V$ characteristics at room temperature in general, or the turn-on bias of $\approx 4 \mathrm{~V}$, the devices bombarded at higher doses required a substantially larger bias before any significant current would flow. At the highest dose of $6 \times 10^{13} \mathrm{~cm}^{-2}$, the turn-on voltage of $\approx 10 \mathrm{~V}$ was much higher than needed to produce high currents in the non-bombarded inner ridge of a weakly-index-guided device. The pulsed $L-I$ characteristics shown in Fig. 2(b) indicate that the threshold current density for lasing increased with increasing IB dose, which is consistent with a higher density of SRH recombination centers. Note that the IB had no appreciable effect on the differential slope efficiency above threshold. The ridge with the highest IB dose did not lase at any measured current density.
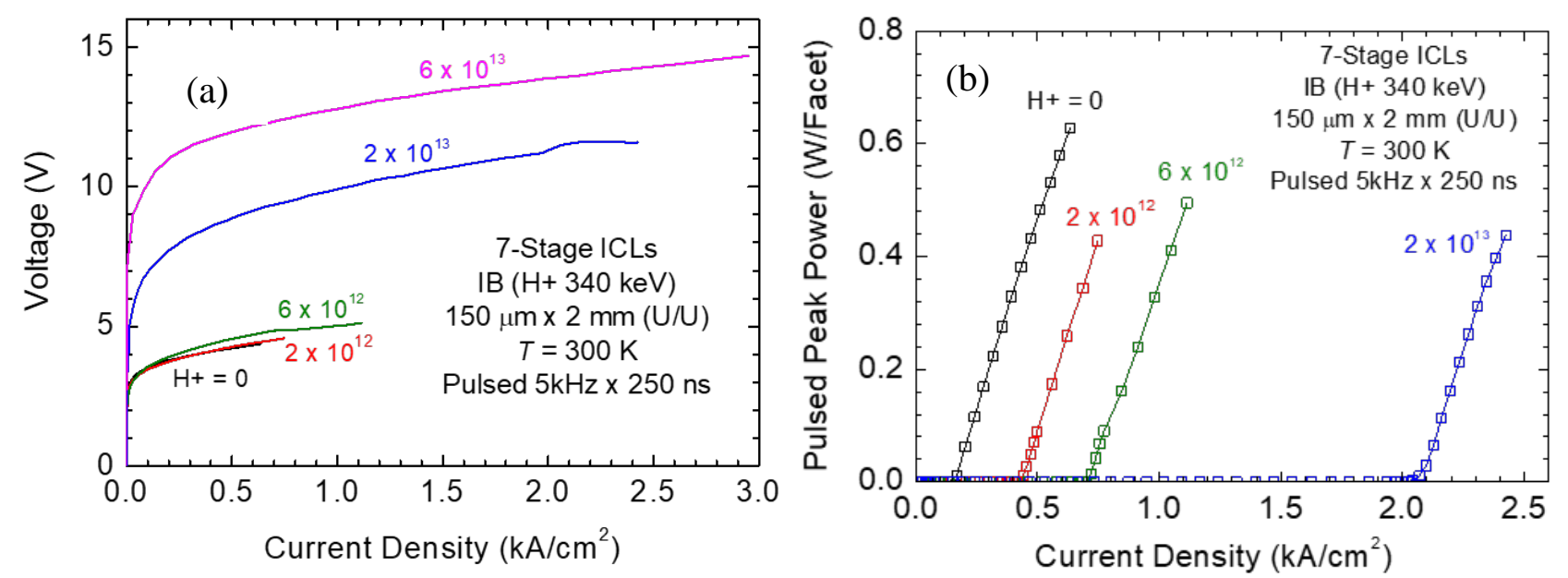

Figure 2 - (a) $I-V$ characteristics of broad-area ICLs that were ion bombarded by $340 \mathrm{keV}$ protons at doses ranging from zero to $6 \times 10^{13} \mathrm{~cm}^{-2}$. (b) $L-I$ characteristics of the same broad-area lasers following IB.

Encouraged by this finding, a weakly-index-guided ICL was fabricated, although instead of employing current injection from the top, as illustrated in Fig. 1(c), the injection was lateral from contacts on both sides of the ridge as illustrated in Fig. 3. This structure was grown with no top cladding layer, and instead a thin layer of Ge was deposited on top to form an inner ridge that confined the fundamental optical mode [37]. The outer ridge was irradiated with $125 \mathrm{keV}$ protons at a dose of $4 \times 10^{13} \mathrm{~cm}^{-2}$, while the inner ridge was protected from the IB by a thick layer of photoresist. By comparing the $L-I-V$ characteristics for devices with different Ge inner ridge widths, ranging nominally from 5 to $15 \mu \mathrm{m}$, it was determined that at threshold the leakage current density into the outer ridge was $\approx 25 \mathrm{~A} / \mathrm{cm}^{2}$. However, the threshold was high and the efficiency very low, due to an unexpected optical loss mechanism that has not been identified. 


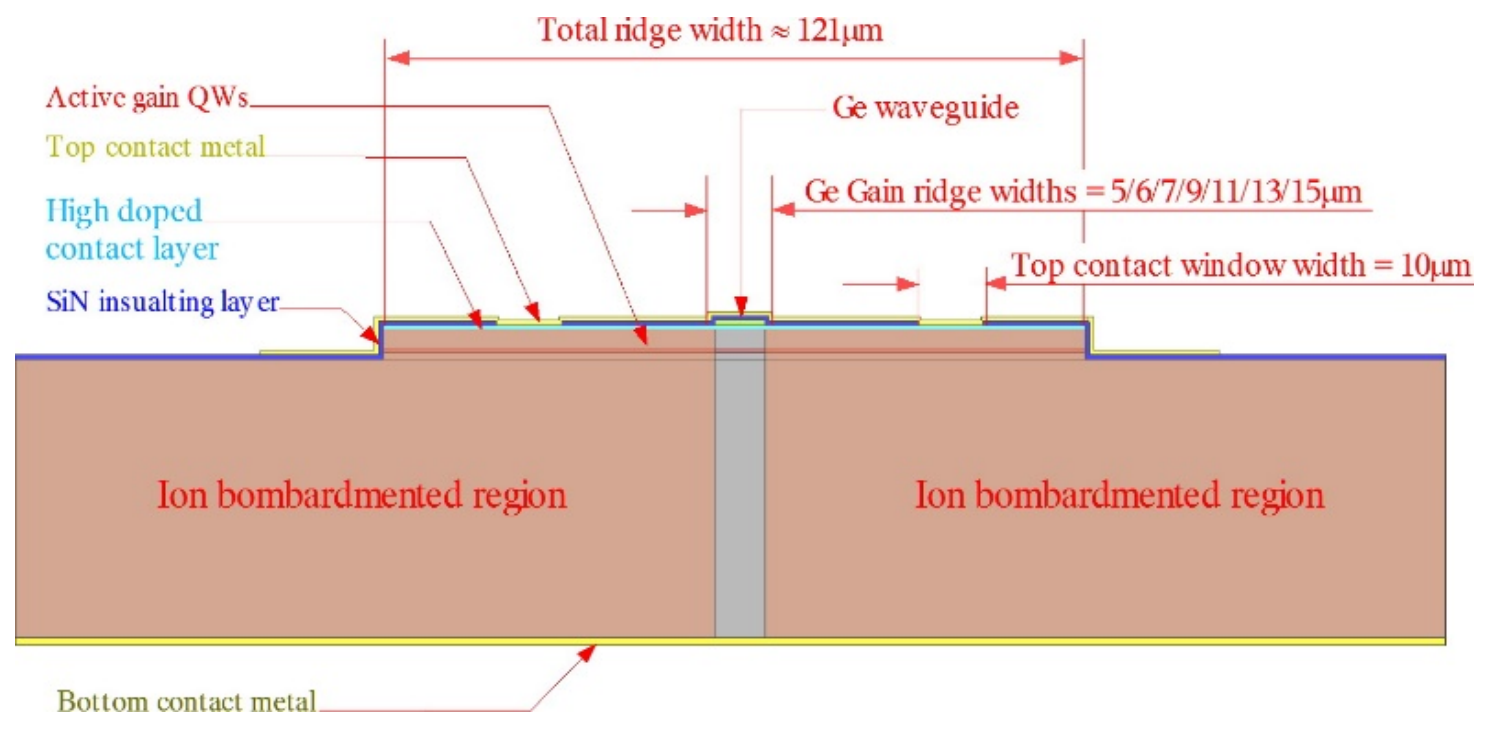

Figure 3. Schematic illustration of a weakly-index-guided ICL ridge with no top cladding layer and a Ge waveguide layer on the top of the ridge. The current injection is lateral, from contacts at the sides of the ridge, and the outer ridge is ion bombarded to suppress current spreading.

\section{ION BOMBARDMENT OF INTERBAND CASCADE VCSELS}

If IB can controllably suppress the spread of current in ICLs, it will also be valuable in other device geometries such as interband cascade vertical cavity surface emitting lasers (ICVCSELs) [17]. Figure 4(a) illustrates that the emitting mesa could then be large enough to contact with an annular ring of metal, by selectively irradiating the outer portion of the mesa while allowing current to flow only into a central circular aperture small enough to support a single lasing mode. The metal must be separated from the lasing area to prevent high scattering loss. To explore the effects of IB on this configuration, $I-V$ characteristics were obtained for ICVCSEL structures in which 10 or 15 active stages (in groups of 5, positioned at the antinodes of the vertical optical mode) were grown above highly-reflective GaSb/AlAsSb Bragg mirrors. No top mirrors were implemented for these transport test structures. The inner apertures were masked while the outer portions of each mesa was irradiated with protons having a series of energies (e.g., $50-250 \mathrm{keV}$ ) and doses (e.g., ranging from $7 \times 10^{12}$ to $6 \times 10^{13} \mathrm{~cm}^{-2}$ ), designed to ensure a uniform defect density through the active core of the structure.

Figure 4(b) plots the $I-V$ characteristics of 10-stage ICVCSEL structures that were processed with mesa diameters ranging from 240 to $540 \mu \mathrm{m}$. These were fully-bombarded (black curves), not bombarded (blue), or bombarded only outside the inner aperture with diameter 280 or $320 \mu \mathrm{m}$ (these dimensions are naturally much larger than would be suitable for a single-mode ICVCSEL). We find that the vertical current flow is again suppressed in the bombarded regions, although there is considerable variation in the degree of leakage. At the expected threshold voltage of $\approx 8 \mathrm{~V}$, where the current density in the non-bombarded structure was $\approx 340 \mathrm{~A} / \mathrm{cm}^{2}$, the leakage current densities in the two fully-bombarded structures were 50 and $100 \mathrm{~A} / \mathrm{cm}^{2}$, i.e., too large to provide meaningful suppression of the current spreading. On the other hand, assuming the same flow of current through the non-bombarded aperture of the two partially-bombarded devices, the $I-V$ characteristics of those devices imply hardly any leakage through the bombarded outer regions. Several other devices in the same series, both non-bombarded and partially bombarded, showed somewhat higher leakage. Structures processed from a 15-stage ICVCSEL wafer exhibited similarly-variable characteristics, which implied leakage currents ranging roughly from 20 to $100 \mathrm{~A} / \mathrm{cm}^{2}$. We conclude that IB can sometimes effectively suppress current spreading into the bombarded regions, at a level that may be useful in devices. But the variability and yield in these preliminary experiments are unacceptable for a practical technology. 
(a)

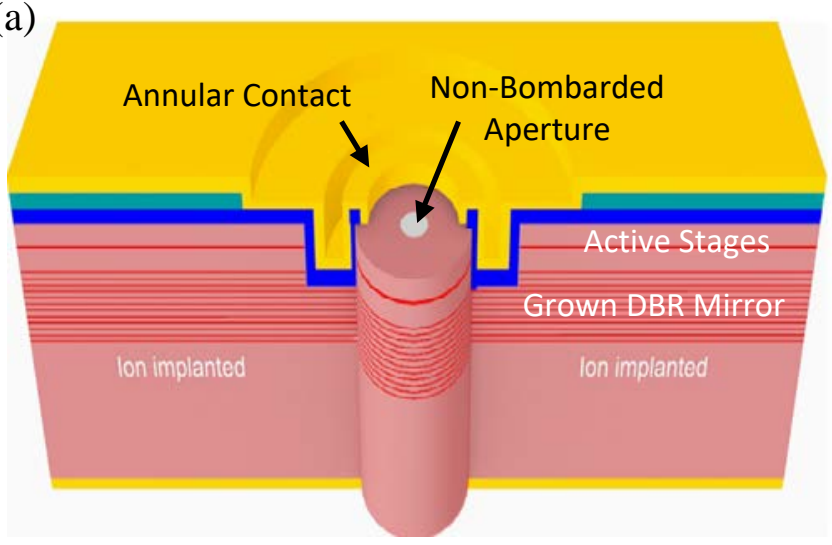

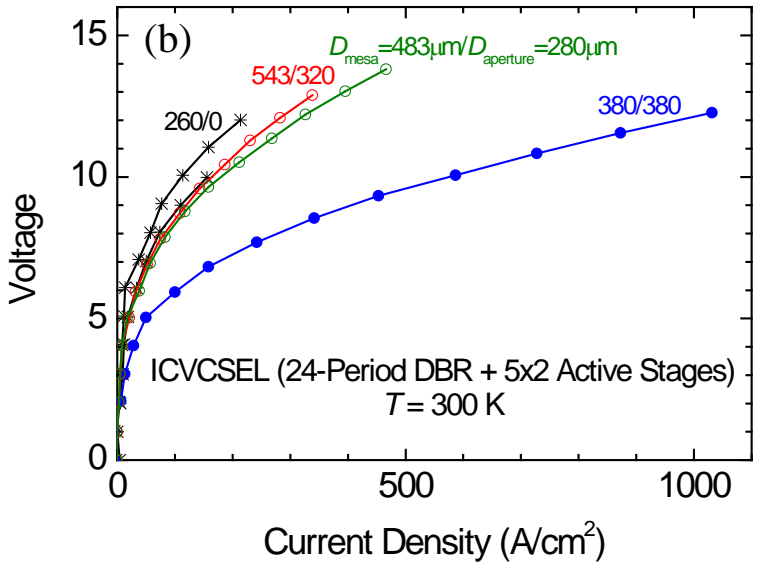

Figure 4. (a) Schematic of an ICVCSEL structure with a GaSb/AlAsSb DBR mirror grown below the active stages (but no top mirror). The outer portion of the mesa is bombarded with protons, while a thick layer of photoresist masks the inner aperture from the irradiation. (b) Vertical $J$ - $V$ characteristics of mesas processed from a 10-stage ICVCSEL structure. Two of the structures (labeled 260/0, black curves) were fully bombarded (no aperture), two were bombarded outside the masked central aperture (red and green), and one was not bombarded (blue). All current densities are normalized to the full mesa area.

\section{ION BOMBARDMENT OF ICL SUB-COMPONENTS}

In those structures for which the vertical flow of current was impeded by IB, three regions seem the most likely candidates for where that may have occurred: (1) the top and bottom InAs-AlSb superlattice cladding layers, (2) the top and bottom lightly-n-doped GaSb separate confinement layers (SCLs), and (3) the active gain stages. In order to isolate the region dominated the current suppression, we performed lateral and vertical transport measurements on bombarded and nonbombarded samples containing only the relevant sub-regions. Lateral resistivities were evaluated by the transition line measurement (TLM) technique [38], which probes top contacts that are separated by varying distances. In most of the structures measured by TLM, an AlAsSb barrier prevented current from reaching the $p$-GaSb substrate. When applied to $n$-GaSb with a low doping level similar to that in the SCLs of an ICL, IB increased the lateral resistance by over a factor of two. Since this binary semiconductor is electrically isotropic, the resistivity along the vertical axis should be the same. Nonetheless, the value of $\rho \approx 0.24 \Omega \mathrm{cm}$ obtained from the fit following IB is far too low to account for the strong suppression of vertical current in some of the devices. It is not surprising that TLM measurements on heavily-doped $n$ GaSb showed a much smaller increase (3\%) of its already-low resistivity.

TLM measurements on a structure with only an InAs/AlSb superlattice similar to the ICL cladding layers showed a negligible increase of the resistivity, to $1.33 \Omega \mathrm{cm}$. However, since this structure is electrically anisotropic the finding does not rule out the possibility of a larger effect along the growth axis. When active stages were tested by TLM, IB actually decreased the resistivity by $10-20 \%$. However, this is even less likely to be indicative of the effect on vertical transport, since the active stages are anisotropic and also divided into sub-regions (electron injector, hole injector, active QWs, etc.) that may react differently to the IB. Measurements on a structure that contained both active stages and a top cladding layer showed $\approx 15 \%$ increase in the lateral resistivity following IB.

Vertical transport measurements were also performed on the same sub-components of the ICL structure. For example, measurements were performed on a 1- $\mu$ m-thick not-intentionally-doped $p$-GaSb epilayer that was grown on a $p$-type GaSb substrate. Although the effect of IB on the epilayer was difficult to quantify precisely because the vertical transport path included the $p$-GaSb substrate which dominated the net electrical resistance, bombarded samples systematically exhibited $13 \%$ higher resistance than non-bombarded control samples processed from the same wafer. When the measurements were repeated following removal of the epilayer by polishing, the vertical resistances of samples that had and had not been irradiated agreed to within $<1 \%$. When measurements were repeated on a sample that was intentionally $p$-doped with Be to a higher level of $5 \times 10^{17} \mathrm{~cm}^{-3}$, the average increase was $2.5 \%$. The effect of IB on $p$-GaSb could potentially be relevant because the GaSb quantum wells forming the hole injector of an ICL could create a bottleneck to electrical transport if IB efficiently traps the holes. 
InAs/AlSb superlattices with layer thicknesses $(24 \AA / 23 \AA)$ and $n$-doped at $7.5 \times 10^{16} \mathrm{~cm}^{-3}$ are typically employed as the top and bottom optical cladding layers in an ICL. A 2- $\mu$ m-thick layer of this material was irradiated with protons having a series of energies ranging from 10 to $325 \mathrm{keV}$ and doses from $4.6 \times 10^{12}$ to $1.1 \times 10^{13} \mathrm{~cm}^{-2}$. The structure also contained an $n^{+}$-InAs top contact layer and InAs/AlSb transition superlattices separating the cladding superlattice from the top contact above and the $n^{+}-\mathrm{GaSb}$ buffer layer and substrate below. Figure 5(a) plots the vertical transport results for 7 bombarded (solid curves) and 7 non-bombarded (dashed curves) ridges $(150 \mu \mathrm{m} \times 2 \mathrm{~mm})$ processed from the same wafer material. The characteristics are seen to be quite consistent from sample to sample. They indicate that at low currents IB significantly increases the voltage dropping across the superlattice, although the ratio of voltages with and without IB decreases somewhat with increasing current density. It falls to $\approx 2.6$ at $50 \mathrm{~A} / \mathrm{cm}^{2}$, at which point Fig. 5(b) shows that the differential resistivity for the four most resistive IB structures is higher by a factor of 1.7. A higher resistivity induced by IB is consistent with a higher threshold voltage for lasing, although the voltage increase indicated here does not appear large enough to fully explain the data in Fig. 1(a). The maximum current density in this experiment, which was limited by the probe station used for the measurements, is well below the lasing threshold.
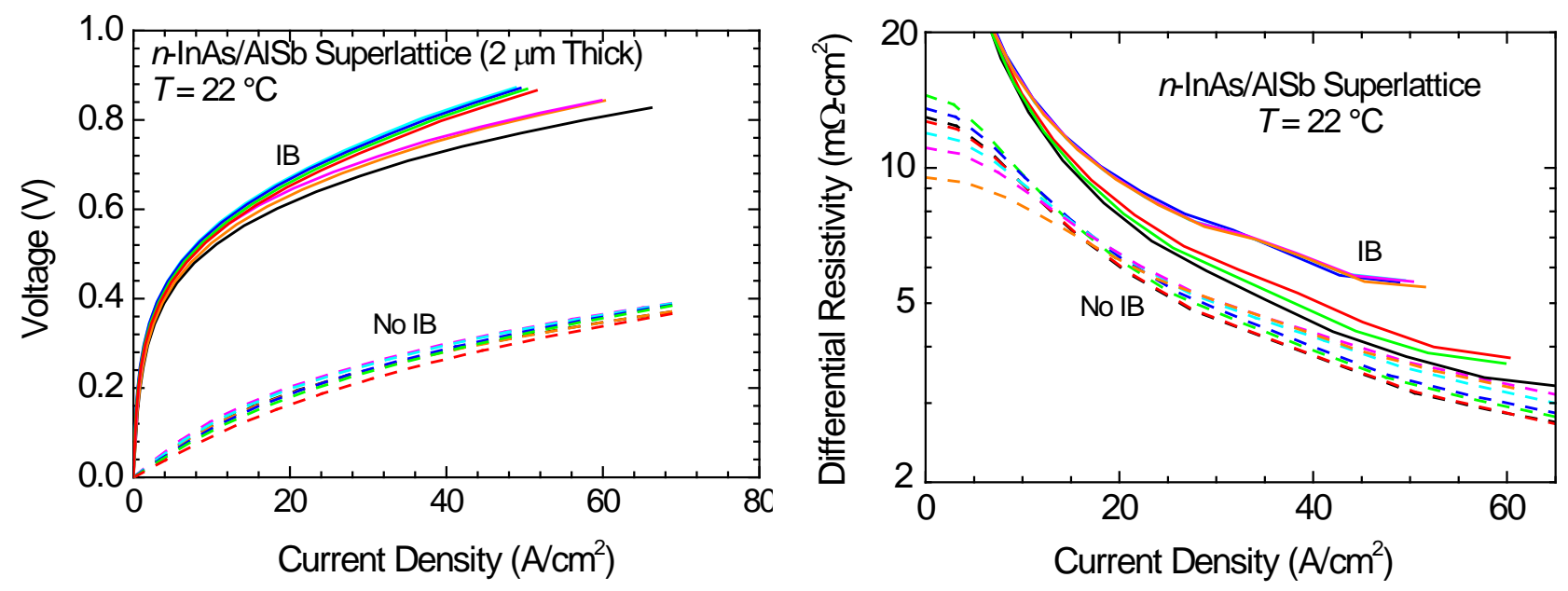

Figure 5. (a) Vertical $I$ - $V$ characteristics of 2 - $\mu \mathrm{m}$-thick bombarded and non-bombarded ridges $(150 \mu \mathrm{m} \times 2$ $\mathrm{mm})$ of $n$-InAs/AlSb superlattices with the same composition $(24 \AA / 23 \AA)$ and doping level $(7.5 \times 1016 \mathrm{~cm}-2)$ typically used as the top and bottom cladding layers in ICLs. Results are shown for 7 bombarded (solid curves) and 7 non-bombarded (dashed curves) devices processed from the same wafer. (b) Differential resistivity corresponding to the same data

Finally, we measured the vertical transport properties of broad-area ridges processed from an interband cascade LED wafer, which contained 22 active stages but no cladding or separate confinement layers. The active stages in this structure are effectively identical to those in an ICL emitting at the same center wavelength. Again 7 bombarded and 7 nonbombarded samples were measured, and again the results from sample to sample were quite consistent as shown in Fig. 6. Surprisingly, in this case the IB devices displayed substantially lower resistivities than the non-bombarded devices. Note also that whereas the $I-V$ characteristics of the non-bombarded devices are quite similar to those of ICLEDs processed from similar material [20], the voltage turn-on is much softer following IB. This appears to reflect damage, possibly associated with leakage due to defect-assisted tunneling between the active electron wells and electron injector in each stage, or some other path. 


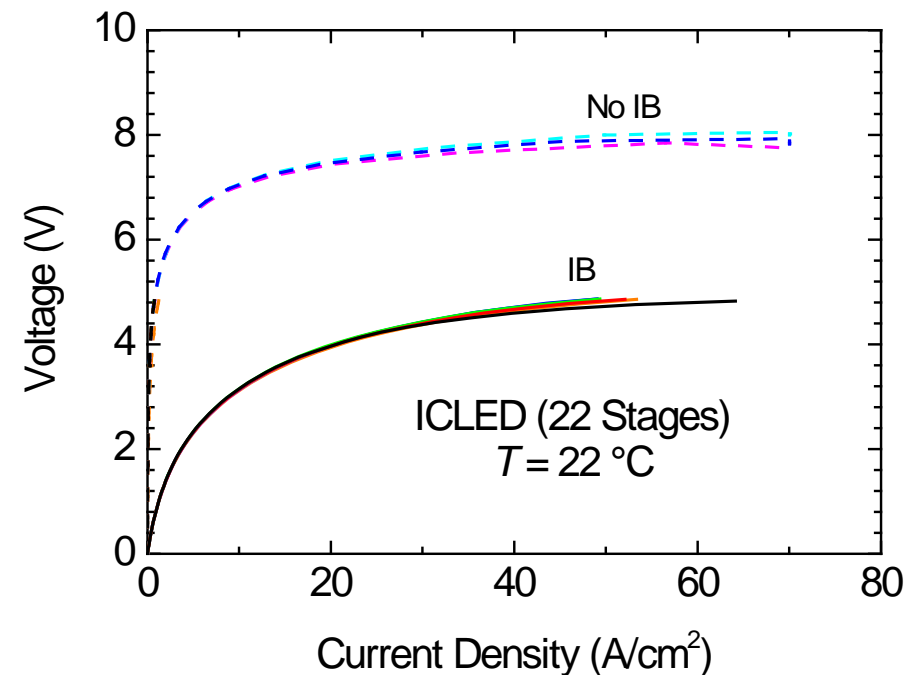

Figure 6. Vertical $I$ - $V$ characteristics of bombarded and non-bombarded 22-stage ICLED ridges $(150 \mu \mathrm{m} \times 2$ $\mathrm{mm}$ ). Results are shown for 7 bombarded (solid curves) and 7 non-bombarded (dashed curves) devices processed from the same wafer.

\section{CONCLUSIONS}

The experiments to date have not conclusively identified the mechanism providing strong suppression of the vertical current flow in some devices, the region where this mechanism occurs, or the reason for inconsistency between the strength (or occurrence at all) of current suppression in different experiments on full ICL structures and constituent components. It is unclear whether the inconsistency is related to subtle differences in the various types of structures, different defect densities in the materials employed, minor differences in the IB recipes (this seems unlikely), or some other random factor in the experiments. Unfortunately, most of the experiments to date have not been repeated on multiple devices processed from multiple wafers, so there has been no opportunity to establish the reproducibility of the findings.

We have, however, established that under some conditions IB can substantially affect the vertical transport in an ICL structure, which if controllable could be valuable when applied to device classes like weakly-index-guided double ridges and mesas patterned for selective current flow in a central emission aperture (e.g., ICVCSELs). In narrowing down the source of strong current suppression in some of the devices, our results imply the effect of IB on $n$-GaSb layers (like the SCLs in an ICL) is measureable but insufficient, on ICLEDs (like the active stages) it decreases rather increases the electrical resistance, and on $n$-InAs/AlSb superlattices (like the ICL cladding layers) it is appreciable but not necessarily sufficient. Nonetheless, the superlattice cladding layers appear the most promising candidates when the results discussed above are taken at face value. Further progress will naturally require a more systematic understanding of the suppression, so it can be controlled reproducibly in practical device structures.

\section{REFERENCES}

[1] I. Vurgaftman, R. Weih, M. Kamp, J. R. Meyer, C. L. Canedy, C. S. Kim, M. Kim, W. W. Bewley, C. D. Merritt, J. Abell, and S. Höfling, J. Phys. D 48, 123001 (2015).

[2] M. Kim, C. L. Canedy, W. W. Bewley, C. S. Kim, J. R. Lindle, J. Abell, I. Vurgaftman, and J. R. Meyer, Appl. Phys. Lett. 92, 191110 (2008).

[3] W. W. Bewley, C. L. Canedy, C. S. Kim, M. Kim, C. D. Merritt, J. Abell, I. Vurgaftman, and J. R. Meyer Opt. Expr. 20, 20894 (2012).

[4] W. W. Bewley, C. S. Kim, C. L. Canedy, C. D. Merritt, I. Vurgaftman, J. Abell, J. R. Meyer, and M. Kim, Appl. Phys. Lett. 103, 111111 (2013).

[5] M. Kim, W. W. Bewley, C. L. Canedy, C. S. Kim, C. D. Merritt, J. Abell, I. Vurgaftman, and J. R. Meyer, Opt. Express 23, 9664 (2015). 
[6] J. Scheuermann, M. von Edlinger, R. Weih, S. Becker, L. Nähle, M. Fischer, J. Koeth, M. Kamp, and S. Höfling, Proc. SPIE 9855, 98550G (2016).

[7] S. M. S. Rassel, L. Li, Y. Li, R. Q. Yang, J. A. Gupta, X. Wu, and G. C. Aers, Opt. Eng. 57, 011021 (2018).

[8] I. Vurgaftman, W. W. Bewley, C. L. Canedy, C. S. Kim, M. Kim, C. D. Merritt, J. Abell, J. R. Lindle, and J. R. Meyer, Nat. Comm. 2, 585 (2011).

[9] I. Vurgaftman et al., IEEE J. Select. Top. Quantum Electron. 17, 1435 (2011).

[10] nanoplus.com/en/products/distributed-feedback-lasers/distributed-feedback-lasers-3000-nm-6000-nm/

[11] F. Xie, M. Stocker, J. Pham, F. Towner, K. Shen, J. Wang, and K. Lascola, Appl. Phys. Lett. 112, 131102 (2018).

[12] C. S. Kim, M. Kim, W. W. Bewley, J. R. Lindle, C. L. Canedy, J. Abell, I. Vurgaftman, and J. R. Meyer, Appl. Phys. Lett. 95, 231103 (2009).

[13] I. Prokhorov, T. Kluge, and C. Janssen, Sci Rep. 9, 4765 (2019).

[14] T. Milde, M. Hoppe, H. Tatenguem, C. Assmann, W. Schade, and J. Sacher, Appl. Opt. 58, C84 (2019).

[15] J. Wang, G. Wang, T. Tan, G. Zhu, C. Sun, Z. Cao, W. Chen, and X. Gao, Opt. Expr. 27, 9610 (2019).

[16] N. H. Pinkowski, Y. Yang, S. J. Cassady, D. F. Davidson, and R. K. Hanson, Combustion and Flame 208, 15 (2019).

[17] W. W. Bewley, C. L. Canedy, M. W. Warren, C. S. Kim, C. D. Merritt, I. Vurgaftman, J. R. Meyer, and M. Kim, Appl. Phys. Lett. 99, 151108 (2016).

[18] D. A. Diaz Thomas, O. Stepanenko, T. Batte, M. Bahriz, S. Calvez, C. Paranthoen, E. Tournie, G. Almuneau, C. Levallois, A. Baranov, and L. Cerutti, Compound Semiconductor Week (Nara Japan, 2019).

[19] G. K. Veerabathran, S. Sprengel, A. Andrejew, and M.-C. Amann, Appl. Phys. Lett. 110, 071104 (2017).

[20] J. Abell, C. S. Kim, W. W. Bewley, C. D. Merritt, C. L. Canedy, I. Vurgaftman, J. R. Meyer, and M. Kim, Appl. Phys. Lett. 104, 261103 (2014).

[21] C. S. Kim, W. W. Bewley, C. D. Merritt, C. L. Canedy, M. V. Warren, I. Vurgaftman, and J. R. Meyer, Opt. Engr. 57, 011002 (2018).

[22] I. Vurgaftman, C. L. Canedy, C. S. Kim, M. Kim, C. D. Merritt, W. W. Bewley, S. Tomasulo, and J. R. Meyer, Conference on Lasers and Electro-Optics, (10-15 May 2020, San Jose CA).

[23] N. Schäfer, J. Scheuermann, R. Weih, J. Koeth, and S. Höfling, Opt. Engr. 58, 117106 (2019).

[24] M. Bagheri, C. Frez, L, A. Sterczewski, I. Gruidin, M. Fradet, I. Vurgaftman, C. L. Canedy, W. W. Bewley, C. D. Merritt, C. S. Kim, M. Kim, and J. R. Meyer, Scientific Reports 8(1), 3322 (2018).

[25] L. A. Sterczewski, J. Westberg, M. Bagheri, C. Frez, I. Vurgaftman, C. L. Canedy, W. W. Bewley, C. D. Merritt, C. S. Kim, M. Kim, J. R. Meyer, and G. Wysocki, Opt. Lett. 44, 2113 (2019).

[26] B. Schwarz, J. Hillbrand, M. Beiser, A. M.,Andrews, G. Strasser, H. Detz, A. Schade, R. Weih, and S. Höfling, S., Optica 6, 890 (2019).

[27] H. Lotfi, L. Li, S. M. S. Rassel, R. Q. Yang, C. J. Correge, M. B. Johnson, P. R. Larson, and J. A. Gupta, Appl. Phys. Lett. 109, 151111 (2016).

[28] A. Spott, E. J. Stanton, N. Volet, J. D. Peters, J. R. Meyer, and J. E. Bowers, IEEE J. Sel. Topics Quant. Electron. 23, 8200818 (2017).

[29] A. Spott, J. D. Peters, M. L. Davenport, E. J. Stanton, C. D. Merritt, W. W. Bewley, I. Vurgaftman, C. S. Kim, J. R. Meyer, J. D. Kirch, L. J. Mawst, D. Botez, and J. E. Bowers, Optica 5, 996 (2018).

[30] A. Malik, A. Spott, E. J. Stanton, J. D. Peters, L. J. Mawst, D. Botez, J. R. Meyer, and J. E. Bowers, IEEE J. Sel. Topics Quant. Electron. 25, 1502809 (2019).

[31] J. R. Meyer, I. Vurgaftman, C. L. Canedy, W. W. Bewley, C. S. Kim, C. D. Merritt, M. V. Warren, R. J. Weiblen, and M. Kim, U.S. Patent Application 16/509613 (2019), "Highly Stable Semiconductor Laser for III-V and Silicon Photonic Integrated Circuits."

[32] N. K. Dutta, d. P. Wilt, and R. J. Nelson, J. Lightwave Technol. 2, 201 (1984).

[33] F. H. Peters and D. T. Cassidy, J. Opt. Soc. Am. B 8, 99 (1991).

[34] S. Forouhar, C. Borgentun, C. Frez, R. M. Briggs, M. Bagheri, C. L. Canedy, C. S. Kim, M. Kim, W. W. Bewley, C. D. Merritt, J. Abell, I. Vurgaftman, and J. R. Meyer, Appl. Phys. Lett. 105, 051110 (2014).

[35] S. J. Pearton, C. R. Abernathy, M. 8. Panish, R. A. Hamm, and L. M. lunardi, J. Appl Phys. 66, 656 (1989).

[36] J. D. Kirch, H. Kim, C. Boyle, C.-C. Chang, L. J. Mawst, D. Lindberg III, T. Earles, D. Botez, M. Helm, J. von Borany, S. Akhmadaliev, R. Böttger, and C. Reyner, Appl. Phys. Lett. 110, 082102 (2017).

[37] J. R. Meyer, I. Vurgaftman, C. L. Canedy, W. W. Bewley, C. S. Kim, C. D. Merritt, M. V. Warren, and M. Kim, U.S. Patent \#9,960,571, 1 May 2018.

[38] G. K. Reeves and H. B. Harrison, IEEE Electron Device Lett., 3, 111 (1982). 